\title{
PROPERTIES OF $\mathrm{SmT}_{2} \mathrm{Sn}_{2}(\mathrm{~T}=\mathrm{Ni}, \mathrm{Cu})$ PHASES
}

\author{
E.A. Görlich ${ }^{a}$, R. KMieć ${ }^{b}$, K. EAqtKa ${ }^{a}$ AND A. Pacyna ${ }^{b}$ \\ anstitute of Physics, Jagiellonian University \\ Reymonta 4, 30-059 Kraków, Poland \\ ${ }^{b} \mathrm{H}$. Niewodniczański Institute of Nuclear Physics, Kraków, Poland
}

Ternary compounds $\mathrm{SmNi}_{2} \mathrm{Sn}_{2}$ and $\mathrm{SmCu}_{2} \mathrm{Sn}_{2}$ were investigated with the X-ray difraction, magnetic susceptibility and electric resistivity methods, as well as using the ${ }^{119} \mathrm{Sn}$ Mössbauer effect under the applied magnetic field. Magnetic susceptibilities of these compounds show the Curie-Weiss behaviour in the temperature range from $15 \mathrm{~K}$ to $280 \mathrm{~K}$ with the enhanced values of the temperature independent term $\chi_{0}$. Both resistivity and magnetic measurements indicate the occurrence of the magnetic ordering in $\mathrm{SmCu}_{2} \mathbf{S n}_{2}$ below $T_{\mathrm{N}}=8 \mathrm{~K}$. The Mössbauer investigations carried out at $4.2 \mathrm{~K}$ under an applied magnetic field of up $6 \mathrm{~T}$ indicate a more complex magnetic structure and a considerable anisotropy. $\mathrm{SmNi}_{2} \mathrm{Sn}_{2}$ remains paramagnetic down to $5 \mathrm{~K}$.

\section{PACS numbers: 72.15.Eb, $76.80 .+y$}

Samarium intermetallic compounds are potentially interesting due to the interplay of crystalline field interactions, $J$-multiplets mixing and possible instability of the $4 f$-state occupancy. Nevcrtheless, systematic studies of their physical properties are not so intense as for cerium or ytterbium systems. With the X-ray powder diffraction a primitive tetragonal $\mathrm{CaBe}_{2} \mathrm{Ge}_{2}$-type structure (space group $P 4 / n m m)$ for both $\mathrm{SmNi}_{2} \mathrm{Sn}_{2}$ and $\mathrm{SmCu}_{2} \mathrm{Sn}_{2}$ phases was confirmed [1,2]. For the former compound, however, the structure was earlier identified in Ref. [3] as of $\mathrm{CeAl}_{2} \mathrm{Ga}_{2}$-type (more often referred to as a body-centred tetragonal $\mathrm{ThCr}_{2} \mathrm{Si}_{2}$-type, space group $I 4 / \mathrm{mmm}$ ). The two tetragonal structures being quite similar are often encountered in ternary $\mathrm{RT}_{2} \mathrm{X}_{2}$ systems and may be viewed as differing in a sequence of basal planes (perpendicular to the $c$-axis) [4]. The stacking order is $\mathrm{R}-\mathrm{X}-\mathrm{T}-\mathrm{X}-\mathrm{R}-\mathrm{T}-\mathrm{X}-\mathrm{T}-\mathrm{R}$... for the primitive cell and $\mathrm{R}-\mathrm{X}-\mathrm{T}-\mathrm{X}-\mathrm{R}-\mathrm{X}-\mathrm{T}-\mathrm{X}-\mathrm{R}$... in the case of a body-centred type. In the former case it leads to the presence of peaks with an odd sum of $h k l$-indices, i.e. they do not fulfill the condition $h+k+l=2 n$ ( $n$ - integer) and may be difficult to find out if the X-ray scattering factors of $\mathrm{T}$ and $\mathrm{X}$ constituents are similar. While in $\mathrm{ThCr}_{2} \mathrm{Si}_{2}$-type compounds all $\mathrm{X}$-atoms occupy equivalent lattice sites there are two crystallographically different positions of tin in the studied samarium compounds: $\mathrm{Sn} 1$ in 2(c) sites (symmetry $4 \mathrm{~mm}$ ) and Sn2 in 2(b) positions (symmetry $\overline{4} 2 \mathrm{~m}$ ). This is straightforwardly confirmed by the occurrence of two components in the ${ }^{119} \mathrm{Sn}$ Mössbauer spectra at room temperature (Fig. 1). 

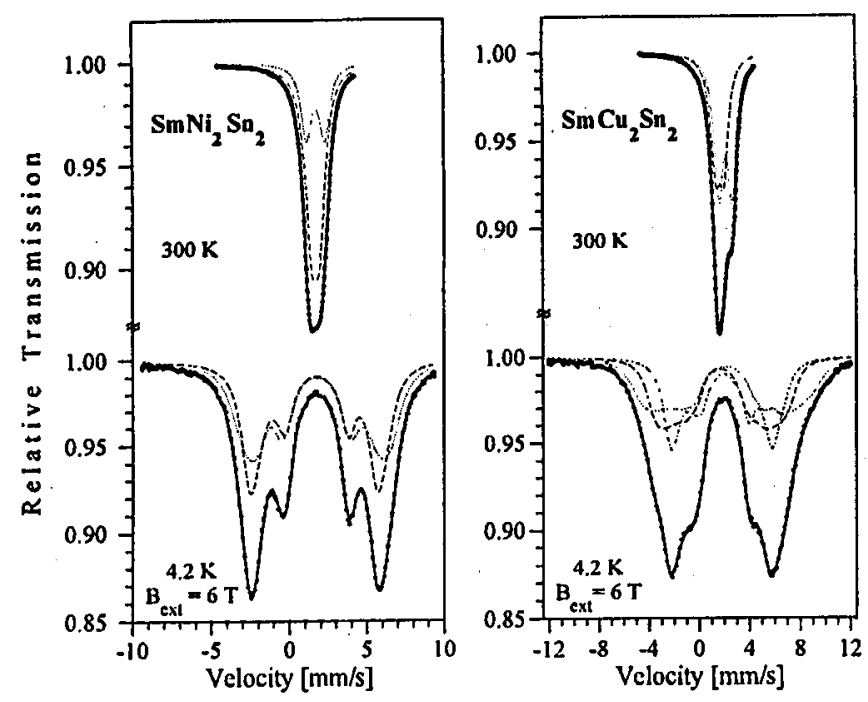

Fig. 1. ${ }^{119} \mathrm{Sn}$ Mössbauer spectra of $\mathrm{SmNi}_{2} \mathrm{Sn}_{2}$ and $\mathrm{SmCu}_{2} \mathrm{Sn}_{2}$ at room temperature and at $4.2 \mathrm{~K}$ under the applied magnetic fields of $6 \mathrm{~T}$.

Magnetic properties of these compounds are determined by samarium ions which are located in 2(c) sites (symmetry $4 \mathrm{~mm}$ ). Magnetic susceptibility measurements have been performed with the Cahn-type balance in weak magnetic fields in a temperature range of $6-280 \mathrm{~K}$. Down to at least $15 \mathrm{~K}$ it follows the Curie-Weiss law with the temperature independent term $\chi_{0}$. The data have been fitted with the expression $\chi(T)=\chi_{0}+C /\left(T-\Theta_{\mathrm{p}}\right)$ and the paramagnetic Curie temperature $\Theta_{\mathrm{p}}$ was found close to $-12.5 \mathrm{~K}$ for both compounds. Because of the close proximity $(\approx 1400 \mathrm{~K})$ of the excited multiplet $\left({ }^{6} \mathrm{H}_{7 / 2}\right)$ to the ground state $\left({ }^{6} H_{5 / 2}\right)$ the $J$-mixing is expected to influence the magnetic behaviour of these compounds along with the crystalline field interactions. The large values of $\chi_{0}, 2.30 \times 10^{-6} \mathrm{~cm}^{3} / \mathrm{g}$ and $2.46 \times 10^{-6} \mathrm{~cm}^{3} / \mathrm{g}$, respectively for nickel and copper phases, are partly due to the Van Vleck temperature-independent paramagnetism. The linear behaviour of $1 / \chi$ (Fig. 2a) remains in contrast to a strongly curved dependence in SmTSn compounds observed by Sakurai et al. [5]. However, Routsi et al. [6] did not report such a concave shape of $1 / \chi$ for one of the latter compounds (SmNiSn). The values of $0.75 \mu_{\mathrm{B}}$ and $0.72 \mu_{\mathrm{B}}$ were obtained for the effective

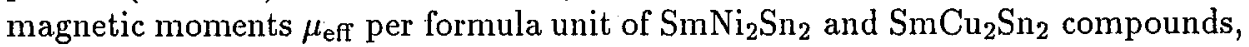
respectively, and are reduced relative to a free trivalent ion ground state value $\left(0.85 \mu_{\mathrm{B}}\right)$.

Both magnetic (Fig. 2a) and electrical resistivity (Fig. 2b) measurements confirm the occurrence of a magnetic ordering in $\mathrm{SmCu}_{2} \mathrm{Sn}_{2}$ below $T_{\mathrm{N}}=8 \mathrm{~K}$, whose presence was already inferred from previous ${ }^{119} \mathrm{Sn}$ Mössbauer experiments [2]. A bend observed at about $55 \mathrm{~K}$ in the temperature dependence of $\mathrm{SmCu}_{2} \mathrm{Sn}_{2}$ electrical resistivity may be due to a phase transition of nonmagnetic character while smooth nonlinearities in $\rho$ vs. $T$ for $\mathrm{SmNi}_{2} \mathrm{Sn}_{2}$ originate clearly from a particular 

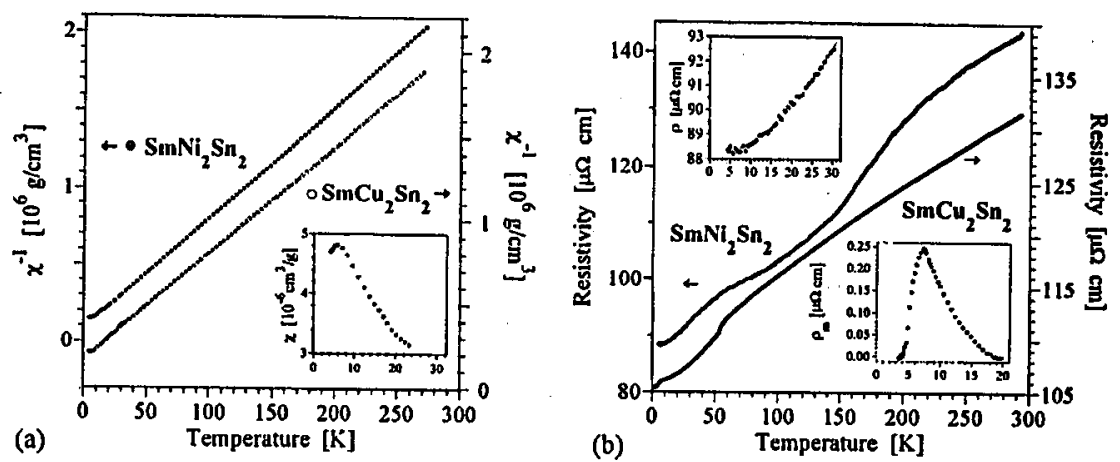

Fig. 2. Temperature dependences of reciprocal magnetic susceptibilities $\chi^{-1}(T)$ (taken at $I=2780 \mathrm{Oc}$ ) (a) and electrical resistivities (b) of $\mathrm{SmNi}_{2} \mathrm{Sn}_{2}$ (full circles) and $\mathrm{SmCu}_{2} \mathrm{Sn}_{2}$ (open circles) compounds. The inset in (a) presents low temperature susceptibility $x(T)$ of $\mathrm{SmCu}_{2} \mathrm{Sn}_{2}$. The insets in (b) depict the low temperature portion of the total resistivity for $\mathrm{SmNi}_{2} \mathrm{Sn}_{2}$ and of the magnetic contribution $\rho_{m}$ for $\mathrm{SmCu}_{2} \mathrm{Sn}_{2}$. The latter quantity, $\rho_{m}$, was obtained by subtracting the resistivity of $\mathrm{LaCu}_{2} \mathrm{Sn}_{2}$ reference while residual resistivities (at lowest temperature of measurement) were assumed equal.

sequence of the crystalline field levels (Fig. 2b). In both cases the features of $\rho(T)$ curves are smeared by a polycrystalline form of the samples as a result of directional averaging of the strongly anisotropic effects. This might be verified with measurements on single crystals.

Thanks to the sensitivity of the nuclear hyperfine interactions to a local enviromment the two crystallographically nonequivalent tin positions in the studied compounds (sites 2(b) and 2(c) in the structure of $P 4 / \mathrm{nmm}$ space group) result in two components present in the room temperature Mössbauer spectra (Fig. 1). The components (depicted with dashed and dotted lines in Fig. 1) differ in their positions (isomer shifts) as well as in the strength of electric quadrupole splittings. The $\gamma$-absorption measurements of magnetic $\mathrm{SmCu}_{2} \mathrm{Sn}_{2}$ sample carried out at $4.2 \mathrm{~K}$ (thus below $T_{\mathrm{N}}=8 \mathrm{~K}$ ) are in favour of three rather than only two components in the spectra. Diamagnetic tin atoms do not effectively contribute to the magnetic field at their nuclei and the hyperfine fields $B_{\mathrm{hf}}$ at ${ }^{119} \mathrm{Sn}$ sites are of transferred character, i.e. arising from the conduction electron polarization as well as from the direct dipolar influence due to a given configuration of samarium magnetic moments. Particular magnetic order sets up three magnetically inequivalent tin sites. Therefore one expects a more complex than the simple (type I [4]) two sublattice antiferromagnetic structure. The presence of three overlapping components does not allow a fully complete separation and deduction of their parameters but with fair reliability (on account of the systematic variation with an increasing external field) the following values of $B_{\mathrm{hf}}: 5.8(1) \mathrm{T}, 2.4(1) \mathrm{T}$ and 1.0(1) $\mathrm{T}$ may be ascribed to the individual subspectra in $\mathrm{SmCu}_{2} \mathrm{Sn}_{2}$ at $4.2 \mathrm{~K}$ (at no external field). Errors given in parenthesis are statistical only and do not reflect uncertainty in component identification. The absorbers consisted of polycrystalline material and the 
application of an external magnetic field $\boldsymbol{B}_{\text {app }}$ resulted in a random orientation of $\boldsymbol{B}_{\text {app }}$ with respect to the local reference system of individual crystallites and thus with respect to the main axis of the electric field gradient at a given tin site. A theoretical description of the spectra required numerical integration over full solid angle. The character of the $\mathrm{SmCu}_{2} \mathrm{Sn}_{2}$ spectra is retained in fields as high as $6 \mathrm{~T}$ (Fig. 1) which makes field-induced phase transitions not feasible in this range. It indicates a high samarium ion anisotropy.

$\mathrm{SmNi}_{2} \mathrm{Sn}_{2}$ remains paramagnetic down to $5 \mathrm{~K}$ but the slight broadening of the ${ }^{119} \mathrm{Sn}$ Mössbauer spectrum at $4.2 \mathrm{~K}$ indicates enhanced samarium moments correlations which may eventually lead to the magnetic ordering at still lower temperatures. At helium temperature and under the applied magnetic field of $6 \mathrm{~T}$ (Fig. 1) the spectrum may be well described by two components and the value of the internal field inferred from the splitting (with the procedure mentioned above) being close to $B_{\text {app }}$.

The authors are grateful to Prof. G.M. Kalvius and Dr. J. Moser of Munich Technical University for the possibility of Mössbauer measurements at applied fields.

\section{References}

[1] R.W. Skolozdra, L.P. Komarovskaya, Fizika Metalov i Melalovedyeneye 53, 1834 (1982) (in Russian).

[2] E.A. Görlich, K. Łątka, J. Moser, Hyp. Int. 50, 723 (1989).

[3] R.V. Skolozdra, W.M. Mandzik, Yu.K. Gorelyenko, W.D. Tkachuk, Fizika Metalov i Metalovedyeneye 52, 966 (1981) (in Russian).

[4] A. Szytuła, in: Handbook of Magnetic Materials, Ed. K.H.J. Buschow, Vol. 6, Ch. 2, Elsevier Science Publishers, Amsterdam 1991, p. 85.

[5] J. Sakurai, K. Kegai, T. Kuwai, Y. Isikawa, K. Nishimura, K. Mori, J. Magn. Magn. Matter. 140-144, 875 (1995).

[6] Ch.D. Routsi, J.K. Yakinthos, H. Gamari-Seale, J. Magn. Magn. Matter. 117, 79 (1992). 